\title{
Graded Hypercapnia-Calibrated BOLD: Beyond the Iso-metabolic Hypercapnic Assumption
}

\author{
Ian D. Driver ${ }^{1 *}$, Richard G. Wise ${ }^{1}$ and Kevin Murphy ${ }^{1,2}$ \\ ${ }^{1}$ Cardiff University Brain Research Imaging Centre, School of Psychology, Cardiff University, Cardiff, United Kingdom, \\ ${ }^{2}$ School of Physics and Astronomy, Cardiff University, Cardiff, United Kingdom
}

\section{OPEN ACCESS}

Edited by:

Federico Giove,

Centro Fermi, Italy

Reviewed by:

Jean Chen,

University of Toronto, Canada

Stefan Posse,

University of New Mexico,

United States

*Correspondence:

lan D. Driver

Driverl@cardiff.ac.uk

Specialty section:

This article was submitted to

Brain Imaging Methods,

a section of the journal

Frontiers in Neuroscience

Received: 31 January 2017

Accepted: 28 April 2017

Published: 18 May 2017

Citation:

Driver ID, Wise RG and Murphy K

(2017) Graded

Hypercapnia-Calibrated BOLD:

Beyond the Iso-metabolic

Hypercapnic Assumption.

Front. Neurosci. 11:276.

doi: 10.3389/fnins.2017.00276
Calibrated BOLD is a promising technique that overcomes the sensitivity of conventional fMRI to the cerebrovascular state; measuring either the basal level, or the task-induced response of cerebral metabolic rate of oxygen consumption $\left(\mathrm{CMRO}_{2}\right)$. The calibrated BOLD method is susceptible to errors in the measurement of the calibration parameter $M$, the theoretical BOLD signal change that would occur if all deoxygenated hemoglobin were removed. The original and most popular method for measuring $M$ uses hypercapnia (an increase in arterial $\mathrm{CO}_{2}$ ), making the assumption that it does not affect $\mathrm{CMRO}_{2}$. This assumption has since been challenged and recent studies have used a corrective term, based on literature values of a reduction in basal $\mathrm{CMRO}_{2}$ with hypercapnia. This is not ideal, as this value may vary across subjects and regions of the brain, and will depend on the level of hypercapnia achieved. Here we propose a new approach, using a graded hypercapnia design and the assumption that $\mathrm{CMRO}_{2}$ changes linearly with hypercapnia level, such that we can measure $M$ without assuming prior knowledge of the scale of $\mathrm{CMRO}_{2}$ change. Through use of a graded hypercapnia gas challenge, we are able to remove the bias caused by a reduction in basal $\mathrm{CMRO}_{2}$ during hypercapnia, whilst simultaneously calculating the dose-wise $\mathrm{CMRO}_{2}$ change with hypercapnia. When compared with assuming no change in $\mathrm{CMRO}_{2}$, this approach resulted in significantly lower $M$-values in both visual and motor cortices, arising from significant dose-dependent hypercapnia reductions in basal $\mathrm{CMRO}_{2}$ of $1.5 \pm 0.6 \% / \mathrm{mmHg}$ (visual) and $1.8 \pm 0.7 \% / \mathrm{mmHg}$ (motor), where $\mathrm{mmHg}$ is the unit change in end-tidal $\mathrm{CO}_{2}$ level. Variability in the basal $\mathrm{CMRO}_{2}$ response to hypercapnia, due to experimental differences and inter-subject variability, is accounted for in this approach, unlike previous correction approaches, which use literature values. By incorporating measurement of, and correction for, the reduction in basal $\mathrm{CMRO}_{2}$ during hypercapnia in the measurement of $M$-values, application of our approach will correct for an overestimation in both $\mathrm{CMRO}_{2}$ task-response values and absolute $\mathrm{CMRO}_{2}$.

Keywords: fMRI, calibrated BOLD, $\mathrm{CMRO}_{2}$, hypercapnia, arterial spin labeling 


\section{INTRODUCTION}

Blood oxygenation level dependent (BOLD) functional MRI (fMRI) signal contrast is widely used as a surrogate measure of underlying neuronal activity (Kwong et al., 1992; Ogawa et al., 1992). The BOLD signal is dependent on the concentration of deoxygenated hemoglobin in blood, which is modulated by changes in cerebral blood flow (CBF), cerebral blood volume $(\mathrm{CBV})$, and cerebral metabolic rate of oxygen consumption $\left(\mathrm{CMRO}_{2}\right)$. Significant inter-region and inter-subject variability in the BOLD response arises due to vascular factors (Chiarelli et al., 2007a; Lu et al., 2008), which could confound interpretation of the underlying neuronal activity from BOLD results. The technique termed calibrated BOLD was developed to address this variability (Davis et al., 1998; Hoge et al., 1999). A calibration parameter $(M)$, defined as the theoretical BOLD signal change that would occur if all deoxygenated hemoglobin were removed, incorporates basal hemodynamic parameters, along with parameters that are dependent on the specific MRI acquisition scheme. This calibration parameter allows for calculation of either absolute $\mathrm{CMRO}_{2}$ (Bulte et al., 2012; Gauthier and Hoge, 2012; Wise et al., 2013), or the task-dependent relative change in $\mathrm{CMRO}_{2}$ (Davis et al., 1998; Hoge et al., 1999). $\mathrm{CMRO}_{2}$ directly reflects tissue metabolism, so is widely considered a more direct measurement of underlying neuronal activity than the cerebrovascular $\mathrm{CBF}$ and $\mathrm{CBV}$ responses. However, this approach is sensitive to errors in the measurement of $M$ (Hoge et al., 1999; Chiarelli et al., 2007b).

The original and most popular method for calculating $M$ uses hypercapnia (an increase in arterial $\mathrm{CO}_{2}$ ). Typically, hypercapnia is presented through inhalation of gas mixtures including $\mathrm{CO}_{2}$, or through reductions in breathing depth and/or pace (e.g., breath hold). The cerebral vasculature is sensitive to changes in partial pressure of carbon dioxide dissolved in arterial blood $\left(\mathrm{PaCO}_{2}\right)$, such that hypercapnia is a potent vasodilator, causing increases in CBF and CBV (Kety and Schmidt, 1948; Ito et al., 2003; Noth et al., 2006; Chen and Pike, 2010b; Ho et al., 2011). For calibrated BOLD, hypercapnia is assumed to be a purely vascular stimulus, providing a change in $\mathrm{CBF}$ and $\mathrm{CBV}$ without a change in $\mathrm{CMRO}_{2}$ (Davis et al., 1998). However, this iso-metabolic assumption is the subject of controversy (Yablonskiy, 2011), with literature indicating increases, decreases and no change in basal $\mathrm{CMRO}_{2}$ with hypercapnia (Kety and Schmidt, 1948; Kliefoth et al., 1979; Rhodes et al., 1981; Hoffman et al., 1982; Horvath et al., 1994; Jones et al., 2005; Sicard and Duong, 2005; Chen and Pike, 2010a; Jain et al., 2011; Xu et al., 2011). Recent human MRI studies (Chen and Pike, 2010a; Jain et al., 2011; Xu et al., 2011) have shown mixed results, calculating $\mathrm{CMRO}_{2}$ using the Fick principle to combine CBF (phase contrast MRI) and OEF (either $\mathrm{T}_{2}$ - or susceptibility-based venous blood oxygenation

Abbreviations: ASL, arterial spin labeling; BOLD, blood oxygenation level dependent (fMRI signal contrast); $\mathrm{CMRO}_{2}$, cerebral metabolic rate of oxygen consumption; CBF, cerebral blood flow; CBV, cerebral blood volume; fMRI, functional magnetic resonance imaging; $\mathrm{PaCO}_{2}$, partial pressure of carbon dioxide dissolved in arterial blood; $\mathrm{P}_{\mathrm{ET}} \mathrm{CO}_{2}$, end-tidal partial pressure of carbon dioxide; ROI, region of interest. measurements). Xu et al. found a significant $13.4 \pm 2.3 \%$ decrease with a $5 \% \mathrm{CO}_{2}$ hypercapnic challenge, whilst Chen and Pike and Jain et al. found no significant change in basal $\mathrm{CMRO}_{2}$ with hypercapnia. Whilst these contrasting findings may arise due to methodological differences, any $\mathrm{CMRO}_{2}$ change with hypercapnia appears to be on the order of the measurement accuracy of these methods. Despite this limited sensitivity, recent calibrated BOLD studies have begun to correct for an assumed reduction in basal $\mathrm{CMRO}_{2}$ with hypercapnia (Bulte et al., 2012), based on these literature values (Xu et al., 2011). An appropriate choice of $\mathrm{CMRO}_{2}$ response (or lack thereof) to hypercapnia is important for calibrated BOLD experiments due to the sensitivity of the method to propagation of errors in $\mathrm{M}$ through to the endpoint $\mathrm{CMRO}_{2}$ calculation (Hoge et al., 1999; Chiarelli et al., 2007b; Blockley et al., 2015).

With measurement sensitivity on the order of the changes observed, current MR techniques are unsuited to fully characterize the relationship between $\mathrm{PaCO}_{2}$ and $\mathrm{CMRO}_{2}$. However, electrophysiological measurements appear to have more sensitivity to investigate the dynamic range of mild hypercapnia $\mathrm{PaCO}_{2}$ values. Studies in both non-human primates and, more recently, in humans show reductions in spontaneous neuronal oscillatory power with hypercapnia (Jones et al., 2005; Zappe et al., 2008; Hall et al., 2011; Xu et al., 2011). We have recently observed a linear relationship between $\mathrm{PaCO}_{2}$ and spontaneous neuronal oscillatory power (Driver et al., 2016). Whilst there is currently no direct relationship established between $\mathrm{CMRO}_{2}$ and neuronal oscillatory power, they may share similar underlying neurochemical mechanisms for their responses to hypercapnia, specifically extracellular $\mathrm{pH}$ changes modulating ATP channels (Dulla et al., 2005). Therefore, in the following paragraphs, we explore the addition to the calibrated BOLD technique of an assumption of a linear relationship between hypercapnia level and $\mathrm{CMRO}_{2}$.

In this work, we present a new approach, relaxing the iso-metabolic hypercapnia assumption in the calibrated BOLD technique. By acquiring multiple levels of hypercapnia, $M$ and the dose-wise $\mathrm{CMRO}_{2}$ response to hypercapnia are solved for as two unknowns in a set of simultaneous equations (one equation for each hypercapnia level). We apply this approach to data presented previously (Murphy et al., 2013), measuring both the calibration parameter $M$ and the dose-wise $\mathrm{CMRO}_{2}$ response to a graded hypercapnia challenge.

\section{MATERIALS AND METHODS}

\section{Theory}

The calibrated BOLD equation, as proposed by Davis et al. (1998) and elegantly restated by Hoge et al. (1999) can be used to model the BOLD signal change during hypercapnia:

$$
\frac{\triangle B O L D_{H C}}{B O L D_{0}}=M\left[1-\left(\frac{C B F_{H C}}{C B F_{0}}\right)^{\alpha-\beta} \cdot\left(\frac{C M R O_{2, H C}}{C M R O_{2,0}}\right)^{\beta}\right]
$$

Where $M$ is the calibration parameter, $\Delta$ denotes the difference between the current state and baseline state, subscripts $H C$ and 0 denote hypercapnia and baseline (normocapnia) conditions, 
respectively. The exponent $\alpha$ describes an assumed coupling relationship between CBF and CBV (Grubb et al., 1974; Chen and Pike, 2010b), whilst $\beta$ is a power-law relationship between venous blood oxygenation and transverse relaxation rate (Ogawa et al., 1993; Boxerman et al., 1995; Driver et al., 2010; Croal et al., 2017). If the iso-metabolic assumption were used, the $\mathrm{CMRO}_{2}$ term would reduce to 1 , with numerator and denominator being equal. $M$ could then be calculated from Equation (1) using measured values for the relative changes in BOLD and CBF due to hypercapnia.

We propose to remove the iso-metabolic assumption, such that the $\mathrm{CMRO}_{2}$ term becomes an unknown parameter to be solved alongside $M$. To do this, we measure BOLD and CBF responses to two levels of hypercapnia, setting up two versions of Equation (1). Since the $\mathrm{CMRO}_{2}$ term may change between the two equations, we assume a linear relationship between $\mathrm{CMRO}_{2}$ and hypercapnia level. A new parameter $\kappa$ is defined as the dose-wise fractional $\mathrm{CMRO}_{2}$ change to a unit $(1 \mathrm{mmHg})$ change in end-tidal partial pressure of carbon dioxide $\left(\mathrm{P}_{\mathrm{ET}} \mathrm{CO}_{2}\right)$, a surrogate measure for $\mathrm{PaCO}_{2}$.

$$
\frac{\mathrm{CMRO}_{2, \mathrm{HC}}}{\mathrm{CMRO}_{2,0}}=1+\kappa \cdot \Delta \mathrm{P}_{\mathrm{ET}} \mathrm{CO}_{2}
$$

Substituting Equation 2 into Equation 1:

$$
\frac{\triangle B O L D_{H C}}{B O L D_{0}}=M\left[1-\left(\frac{C B F_{H C}}{C B F_{0}}\right)^{\alpha-\beta} \cdot\left(1+\kappa \cdot \Delta P_{E T} C O_{2}\right)^{\beta}\right]
$$

Therefore, with $\mathrm{P}_{\mathrm{ET}} \mathrm{CO}_{2}$ measured by sampling exhaled gas, $M$ and $\kappa$ are two unknowns, which can be solved for using two equations, one for each hypercapnia level.

\section{Data Acquisition}

Fifteen subjects (7M/8F, age range 21-36 years) participated in 2 sessions in which scans were acquired using a $3 \mathrm{~T}$ whole body MRI system (GE Excite HDx, Milwaukee, WI, USA) with an eight-channel receive coil. The School of Psychology, Cardiff University Ethics Committee approved this study and subjects gave written informed consent prior to participating.

Data were acquired using a pulsed arterial spin labeling (ASL) proximal inversion and control for off-resonance effects (PICORE), quantitative imaging of perfusion using a single subtraction (PICORE QUIPSS II) (Wong et al., 1998) imaging sequence. This sequence used a dual-echo gradient echo readout (Liu et al., 2002) and spiral-out k-space acquisition [Glover, 1999; 490 repetitions (image volumes), TE1 $=3.3 \mathrm{~ms}$ TE2 $=29 \mathrm{~ms}$, $\mathrm{TR}=2,200 \mathrm{~ms}$, flip angle $90^{\circ}$, FOV $22 \mathrm{~cm}$, matrix $64 \times 64,12$ slices of $7 \mathrm{~mm}$ thickness with an inter-slice gap of $1 \mathrm{~mm}$, TI1 $=600 \mathrm{~ms}$, TI $2=1,500 \mathrm{~ms}$ for the most proximal slice, $10 \mathrm{~cm}$ inversion slab thickness, adiabatic hyperbolic secant inversion pulse, $10 \mathrm{~mm}$ gap between labeling slab and bottom slice, $10 \mathrm{~cm}$ QUIPSS II saturation band thickness]. Additionally, whole brain $\mathrm{T}_{1}$-weighted structural scan (fast spoiled gradient recalled echo, $1 \times 1 \times 1 \mathrm{~mm}$ voxels, TI/TR/TE $=450 / 7.8 / 3 \mathrm{~ms}$ ) was acquired for segmentation of gray matter.
Participants were presented with hypercapnia levels of +4 and $+8 \mathrm{mmHg} \Delta \mathrm{P}_{\mathrm{ET}} \mathrm{CO}_{2}$ above their normal resting level. End-tidal $\mathrm{CO}_{2}$ levels were changed at 2-min intervals between baseline, +4 and $+8 \mathrm{mmHg}$ values, in a randomized order. This provided three 2-min blocks for each condition, across the $18 \mathrm{~min}$ ASL scan. Gas mixtures were delivered to the subject through a tight-fitting face-mask (Quadralite, Intersurgical, Wokingham, Berkshire, UK). Flow rates of two gas mixtures, namely medical air $\left(21 \% \mathrm{O}_{2}, 79 \% \mathrm{~N}_{2}\right)$ and a $5 \% \mathrm{CO}_{2}$ mixture $\left(5 \% \mathrm{CO}_{2}, 20 \% \mathrm{O}_{2}, 75 \% \mathrm{~N}_{2}\right)$, were manually adjusted to provide an inspired gas mixture of $30 \mathrm{~L} / \mathrm{min}$. The respiratory circuit included a reservoir on the expired limb to permit re-breathing in the event that the instantaneous inspiratory rate exceeded $30 \mathrm{~L} / \mathrm{min}$. Expired gas concentrations were sampled from the face-mask and $\mathrm{P}_{\mathrm{ET}} \mathrm{CO}_{2}$ and $\mathrm{P}_{\mathrm{ET}} \mathrm{O}_{2}$ (end-tidal $\mathrm{pO}_{2}$ ) were measured using rapidly responding gas analyzers (AEI Technologies, Pittsburgh, PA, USA). A manual feedback procedure was used to reach each hypercapnia level, whereby the respective flow rates of medical air and the $5 \% \mathrm{CO}_{2}$ mixture were adjusted to reach the $\mathrm{P}_{\mathrm{ET}} \mathrm{CO}_{2}$ target.

A combined visual and motor task was simultaneously performed, consisting of blocks of $8 \mathrm{~Hz}$ flashing checkboard and right-handed self-paced finger tapping with a range of block durations between 20 and $30 \mathrm{~s}$. These blocks were interspersed with 20-30 s blocks of rest. The range in task and rest block durations were chosen so that the visual, motor and $\mathrm{CO}_{2}$ tasks had minimal correlation with each other. The task data was used to define primary visual and motor cortex ROIs.

\section{Data Analysis}

$\mathrm{CBF}$ time series were calculated from the first echo by separating tag and control time series, interpolating to the TR and subtracting. A similar procedure using averaging rather than subtraction yielded BOLD time series from the second echo. $\mathrm{R}_{2}{ }^{*}$ was also calculated by performing an exponential fit across the two echo times, separately for tag and control time series, then combining tag and control $\mathrm{R}_{2}$ * values by surround averaging. A gray matter (GM) ROI was calculated for each subject by segmenting their anatomical image into three tissue types (gray matter, white matter and cerebrospinal fluid) using FSL's fast. The GM map was resampled to the functional data resolution. Visual and motor ROIs were calculated by including $\mathrm{CO}_{2}$, visual and motor timings in a voxel-wise GLM for both the BOLD and $\mathrm{CBF}$ data. The results for each subject were transformed into MNI space and a voxel-wise $t$-test against 0 across subject was performed. The $t$-test maps were FDR thresholded at $p$ $=0.05$. The resulting BOLD and CBF activation maps were transformed back into individual subject space. The visual and motor ROIs were then calculated for each subject by taking an intersection map between the BOLD activation map, the $\mathrm{CBF}$ activation map and the individual GM ROI. Therefore, a voxel was only included in the motor ROI if it significantly responded to the motor task in both the BOLD and CBF data and was present in the individual's GM mask. A similar procedure was used for the visual ROI. Once the ROIs were defined, the BOLD and CBF time series were averaged over the visual, 
motor, and GM ROIs, then linear detrending was performed using baseline periods, before averaging across sessions for each subject.

BOLD and CBF responses to each hypercapnia level (relative to baseline) were input into +4 and $+8 \mathrm{mmHg}$ versions of Equation (3), then these two equations were solved simultaneously for $M$ and $\kappa$, using a two-parameter non-linear fitting routine (Isqcurvefit, Matlab, The MathWorks, Natick, USA). Subjects that reached the boundary conditions of the non-linear fitting routine were removed from further analysis (boundary conditions $1<\mathrm{M}<20 \%$; $-5<\kappa<+5 \% / \mathrm{mmHg}$ ). For comparison with the iso-metabolic assumption, the same two equations as above ( +4 and $+8 \mathrm{mmHg}$ versions of Equation 3) were solved simultaneously using a one-parameter fit, to calculate $\mathrm{M}$ whilst fixing $\kappa=0$.

Optimized values of $\alpha=0.14$ and $\beta=0.91$ were used (Griffeth and Buxton, 2011), hereafter referred to as the empirically derived $\alpha / \beta$ pairing. To ensure that our findings were not biased by choice of this $\alpha / \beta$ pairing, we also repeated the non-linear fitting with the following two alternative $\alpha / \beta$ pairings that have been used previously for calibrated BOLD experiments at $3 \mathrm{~T}$. Values of $\alpha=0.2$ and $\beta=1.3$ have been used at $3 \mathrm{~T}$ (Bulte et al., 2012), hereafter referred to as the $3 T$ specific $\alpha / \beta$ pairing. Finally, a simplified model has been proposed recently, substituting $\alpha / \beta$ for a single parameter $\theta=0.06$ at $3 \mathrm{~T}$ (Merola et al., 2016). In this case, this simplified model is equivalent to $\alpha=0.06$ and $\beta=1$, hereafter referred to as the simplified model $\alpha / \beta$ pairing.

\section{RESULTS}

The two levels of hypercapnia resulted in $\triangle \mathrm{P}_{\mathrm{ET}} \mathrm{CO}_{2}$ increases of $4.8 \pm 0.3$ and $8.4 \pm 0.4 \mathrm{mmHg}$ (mean \pm SEM across subjects). BOLD and CBF responses to the two levels of hypercapnia and the respective task (where applicable) are presented for visual, motor cortices and the remaining GM in Table 1. To assess BOLD sensitivity at the $29 \mathrm{~ms}$ TE, baseline $\mathrm{R}_{2}{ }^{*}$ is also reported for each ROI in Table 1. Group-average maps (MNI space) of the BOLD and CBF responses to the two levels of hypercapnia are shown in Figure 1.

For results presented based on either the two- or oneparameter fits, the number of subjects included after discarding those that reached the boundary conditions are presented in the form $(N=\# / 15)$, where \# corresponds to the number of subjects included. The two-parameter fit with the empirically derived $\alpha / \beta$ pairing $(0.14 / 0.91)$ gave $M=9.6 \pm 1.3 \%(N=14 / 15)$ and $M=4.7 \pm 0.6 \%(N=13 / 15)$, in the visual and motor cortices respectively and $M=8.6 \pm 0.9 \%(N=15 / 15)$ in the remaining GM. The dose-dependent hypercapnia $\mathrm{CMRO}_{2}$ parameter $\kappa=-1.5 \pm 0.6 \% / \mathrm{mmHg}, \kappa=-1.8 \pm 0.7 \% / \mathrm{mmHg}$ and $\kappa=-1.3 \pm 0.4 \% / \mathrm{mmHg}$ showed significant reductions in $\mathrm{CMRO}_{2}$ with hypercapnia level (Wilcoxon $p=0.04, p=0.03$, and $p=0.002$ ). The two-parameter fit resulted in significantly lower $M$-values than the one-parameter fit for subjects that did not reach the boundary conditions for both fits [Figure 2; visual $p=0.04,(N=9 / 15)$; motor $p=0.008,(N=13 / 15)$; GM $p=0.001,(N=14 / 15)]$.
TABLE 1 | BOLD and CBF responses (\% change from baseline) for the two hypercapnia levels and for the visual and motor tasks (mean \pm SEM across subjects).

\begin{tabular}{lcccc}
\hline ROI & $\boldsymbol{\Delta} \mathbf{P}_{\mathbf{E T}} \mathbf{C O}_{\mathbf{2}}(\mathbf{m m H g})$ & $\% \mathbf{B O L D}$ & $\% \mathbf{C B F}$ & Baseline $\mathbf{R}_{\mathbf{2}}{ }^{*}\left(\mathbf{s}^{-\mathbf{1}}\right)$ \\
\hline Visual & $4.8 \pm 0.3$ & $0.9 \pm 0.3$ & $13 \pm 3$ & $31.3 \pm 1.6$ \\
& $8.4 \pm 0.4$ & $1.7 \pm 0.3$ & $19 \pm 4$ & \\
& 0 (+ Task) & $1.7 \pm 0.1$ & $33 \pm 3$ & \\
\hline Motor & $4.8 \pm 0.3$ & $0.8 \pm 0.1$ & $30 \pm 15$ & $22.3 \pm 0.7$ \\
& $8.4 \pm 0.4$ & $1.4 \pm 0.2$ & $43 \pm 16$ & \\
\hline \multirow{2}{*}{$\mathrm{GM}$} & 0 (+ Task) & $1.3 \pm 0.1$ & $73 \pm 19$ & \\
& $4.8 \pm 0.3$ & $1.0 \pm 0.1$ & $13 \pm 2$ & $31.7 \pm 0.9$ \\
& $8.4 \pm 0.4$ & $1.6 \pm 0.1$ & $17 \pm 3$ & \\
\hline
\end{tabular}

Baseline $R_{2}{ }^{*}$ values are also presented for each $R O I$.

The effect of changing the $\alpha / \beta$ pairing is assessed in Figures 3, 4. Figure 3 shows a scatter plot of $\kappa$ across subjects for each $\alpha / \beta$ pairing and each region of interest, with mean \pm SEM across subjects presented above. The group-averaged $\kappa$-values remain stable across $\alpha / \beta$ pairings, indicating a robust decrease in $\mathrm{CMRO}_{2}$ during hypercapnia. Figure 4 presents $M$ values across $\alpha / \beta$ pairings. Despite the amplitude of $M$ varying with $\alpha / \beta$ pairing, $M$ calculated using the two-parameter fit was consistently lower than that calculated using the one-parameter (iso-metabolic) fit.

\section{DISCUSSION}

Through use of a graded hypercapnia gas challenge, we are able to remove the bias caused by a reduction in basal $\mathrm{CMRO}_{2}$ during hypercapnia, whilst simultaneously calculating the dosewise $\mathrm{CMRO}_{2}$ change with hypercapnia. We observed consistently lower $M$-values when calculated from our new approach, compared to those calculated using an iso-metabolic hypercapnia assumption, evidence for a systematic overestimation of $M$ when using the iso-metabolic assumption. In terms of studies calculating the relative change in $\mathrm{CMRO}_{2}$ to a task, this overestimation in $M$ would result in an overestimation in the $\mathrm{CMRO}_{2}$ task response (see Equation 1, replacing hypercapnia terms with the equivalent task response terms). In terms of studies investigating absolute $\mathrm{CMRO}_{2}$ measurements, the overestimation in $M$ would lead to an overestimation of absolute $\mathrm{CMRO}_{2}$ (Blockley et al., 2015).

In this study, we define a dose-wise $\mathrm{CMRO}_{2}$ response to hypercapnia, $\kappa$. Our findings suggest a decrease in $\mathrm{CMRO}_{2}$ with hypercapnia in the primary visual and motor cortices, as well as in the remaining GM. The scale of the $\mathrm{CMRO}_{2}$ reduction is broadly similar to that observed by Xu et al. (2011), where their $5 \% \mathrm{CO}_{2}$ challenge resulted in a $13.4 \%$ decrease in global $\mathrm{CMRO}_{2}$. Based on the reported average $\Delta \mathrm{P}_{\mathrm{ET}} \mathrm{CO}_{2}$ of $8.7 \mathrm{mmHg}$, this is equivalent to $\kappa=-1.5 \% / \mathrm{mmHg}$. This is within the range of the GM value that we measured of $\kappa=-1.3 \pm 0.4 \% / \mathrm{mmHg}$. Unlike previous work, which measured the global $\mathrm{CMRO}_{2}$ response 

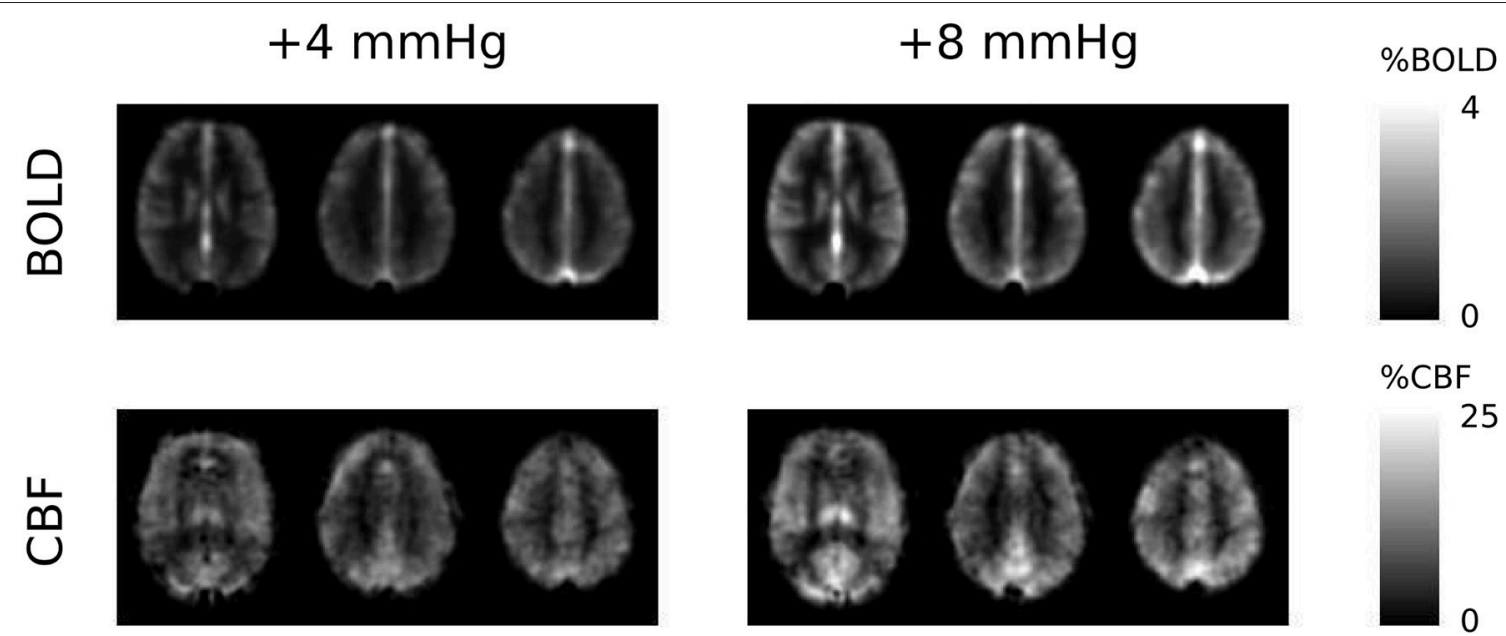

FIGURE 1 | Group-average maps of BOLD and CBF responses to the +4 and $+8 \mathbf{m m H g} \Delta \mathrm{P}_{\mathrm{ET}} \mathrm{CO}_{2}$ hypercapnia conditions. The three slices shown are at the level of $\mathrm{MNI}$ coordinate $\mathrm{Z}=+12,+28$, and $+42 \mathrm{~mm}$, respectively.
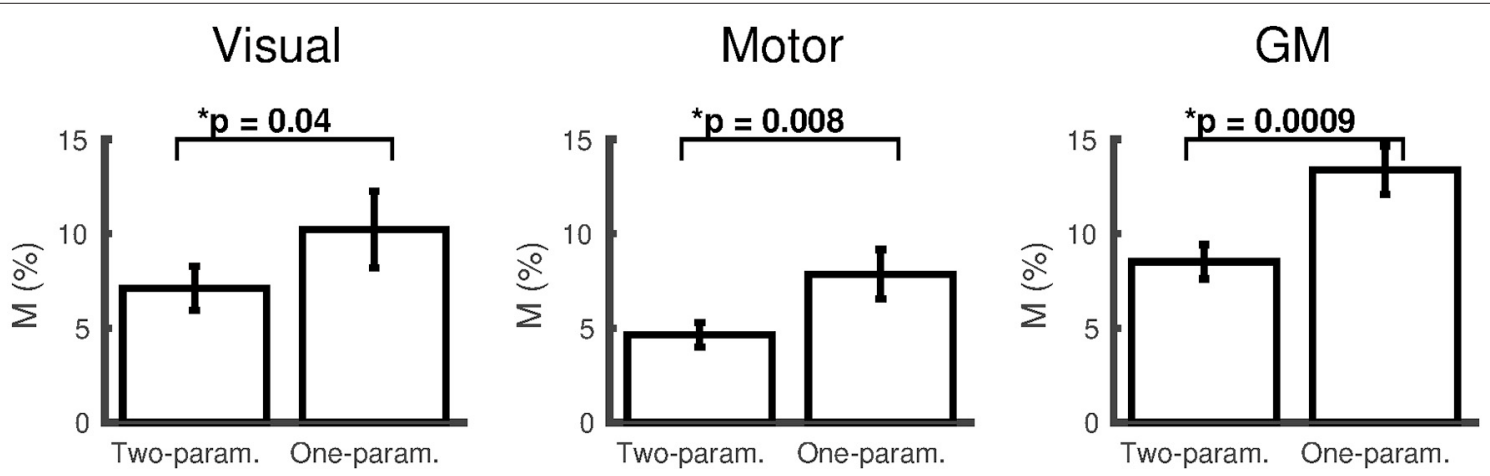

FIGURE 2 | Comparison of $M$ (mean \pm SEM) calculated using the empirically derived $\alpha / \beta$ pairing $(0.14 / 0.91)$ from the two-parameter $\left(\Delta C M R O_{2}\right.$ varies linearly with $\Delta \mathrm{P}_{\mathrm{ET}} \mathrm{CO}_{2}$ ) and one-parameter (iso-metabolic) models for subjects that did not reach the boundary conditions for both fits (visual cortex $N=$ 9; motor cortex $N=13$; remaining GM $N=14)$. ${ }^{*} p<0.05$.

to hypercapnia, our approach can provide measurements that are localized to specific brain regions. Our initial findings hint at some spatial heterogeneity in the $\mathrm{CMRO}_{2}$ response to hypercapnia, with the hypercapnia $\mathrm{CMRO}_{2}$ reduction appearing to be greater in the motor than visual cortex. However, this dataset does not have the sensitivity to resolve whether this is a significant difference (see inter-subject variability in Figure 3). Likewise, the paradigm used here is not optimized for voxelwise mapping of $M$ and $\kappa$, however with a suitably optimized graded hypercapnia paradigm design that enhances voxelwise sensitivity, this approach could be translated to mapping $M$ and $\kappa$, for application in mapping absolute $\mathrm{CMRO}_{2}$. The potential spatial heterogeneity of the $\mathrm{CMRO}_{2}$ response to hypercapnia will be investigated in future studies, incorporating a specifically optimized hypercapnia paradigm and a more sophisticated fitting algorithm (Germuska et al., 2016). This approach for mapping the $\mathrm{CMRO}_{2}$ response to hypercapnia should be considered in the context of a potential alternative, O-15 PET. There is an extensive literature on mapping $\mathrm{CBF}$ and $\mathrm{CMRO}_{2}$ using $\mathrm{O}-15$
PET (e.g., Mintun et al., 1984; Ter-Pogossian and Herscovitch, 1985; Kudomi et al., 2013). Whilst there are many studies to use O-15 PET to measure the CBF response to hypercapnia (e.g., Ito et al., 2003), the O-15 PET literature on mapping the $\mathrm{CMRO}_{2}$ response to hypercapnia is limited, with no change in $\mathrm{CMRO}_{2}$ measured in anesthetized dogs (Rhodes et al., 1981). The steady-state variant of the $\mathrm{CMRO}_{2}$ measurement used in that work may also be biased by not accounting for intravascular ${ }^{15} \mathrm{O}_{2}$ (Lammertsma and Jones, 1983; Lammertsma et al., 1983; Ter-Pogossian and Herscovitch, 1985). Whilst our method is constrained by the accuracy of the assumptions associated with the calibrated BOLD technique (Hoge et al., 1999; Chiarelli et al., 2007b; Chen and Pike, 2010b; Blockley et al., 2015; Croal et al., 2017), it is non-invasive, not requiring use of radioactive tracers. It also has the potential for finer temporal and spatial resolution than O-15 PET.

The approach we present here requires an assumption as to the form of the relationship between $\mathrm{CMRO}_{2}$ and $\mathrm{PaCO}_{2}$; in this case, the assumption is of a linear relationship. Beyond the linear 


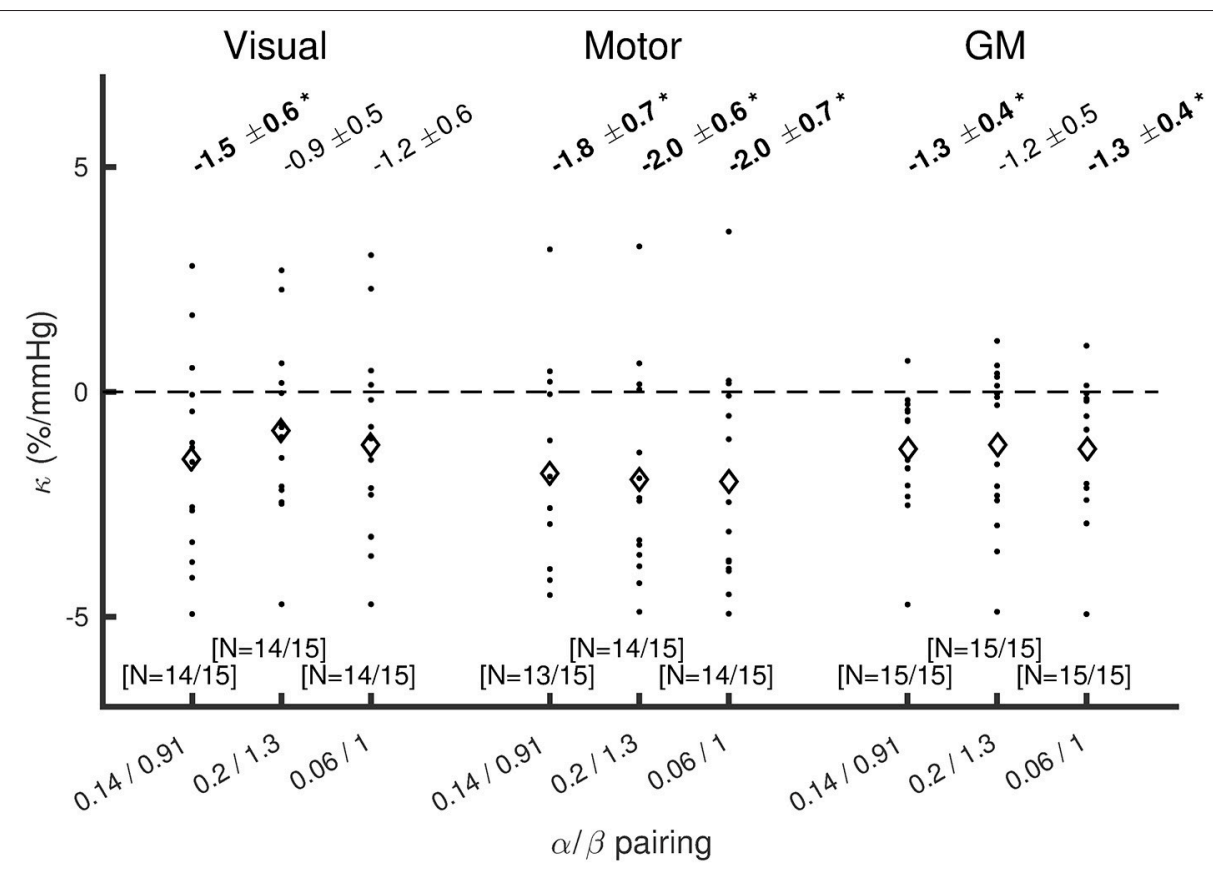

FIGURE 3 | Plot of $\kappa$ across subjects for each $\alpha / \beta$ pairing, for visual cortex, motor cortex, and the remaining GM. Diamonds show mean $\kappa$ across subjects. The values presented at the top are mean \pm SEM across subjects for $\kappa$, with bold text and *indicating $p(\kappa \neq 0)<0.05(\mathrm{Wilcoxon}$ sign rank test). The numbers of subjects included after discarding those that reached the boundary conditions are presented in the form $(N=\# / 15)$ at the bottom of each plot.

relationship, no prior assumption is made as to whether $\mathrm{CMRO}_{2}$ increases, decreases or remains constant with hypercapnia. This linear assumption is also implicitly made as part of the isometabolic assumption, or when using previous literature values for a $\mathrm{CMRO}_{2}$ decrease. The linear relationship between $\mathrm{CMRO}_{2}$ and hypercapnia level is based on our recent observations of a linear relationship between hypercapnia level and spontaneous neuronal oscillatory power (Driver et al., 2016). Whilst there is currently no direct relationship established between $\mathrm{CMRO}_{2}$ and neuronal oscillatory power, they may share similar underlying neurochemical mechanisms for their responses to hypercapnia, specifically extracellular $\mathrm{pH}$ changes modulating ATP channels (Dulla et al., 2005). Even if the relationship includes some nonlinearity, bias introduced by a linear correction will be smaller than the bias from no correction.

The constrained non-linear fitting performed here to solve for $M$ and $\kappa$ imposed boundary conditions on these parameters. These boundary conditions $1<\mathrm{M}<20 \%$; $-5<\kappa<+5 \% / \mathrm{mmHg}$ were chosen to be sufficiently broad to include the range of values that would be reasonably expected when averaging across these regions of interest, based on previous literature (Leontiev and Buxton, 2007; Chiarelli et al., 2007a; Mark et al., 2011; Xu et al., 2011). Therefore, where the fitting algorithms returned values that reached these boundary conditions, this is likely to be due to noise in the data, most likely the ASL data, rather than being an actual physiological outlier. It is for this reason that we chose to discard results where the boundary condition was reached, an approach we have taken previously (Murphy et al., 2013; Wise et al., 2013). Out of the 18 versions of the non-linear fitting (three ROIs over three $\alpha / \beta$ pairings for each of the two- and one-parameter fits), 14 had either one or no subjects reaching the boundary condition. There were 3 occasions where more than two subjects reached the boundary condition, all occurring in the visual ROI for the one-parameter fit, 5 subjects each for the $0.14 / 0.91$ and $0.2 / 1.3 \alpha / \beta$ pairings and 4 subjects for the $0.06 / 1 \alpha / \beta$ pairing. This is consistent with the visual ROI dataset containing more variance than the motor and remaining GM ROIs (see errorbars in Figure 4). Further, a BOLD-weighted second echo time of $29 \mathrm{~ms}$ used here has been optimized previously for BOLD contrast, based on GM-averaged $\mathrm{R}_{2}{ }^{*}$ (Wise et al., 2013; Germuska et al., 2016). The $29 \mathrm{~ms}$ echo time is optimal for BOLD contrast for the $\mathrm{R}_{2}{ }^{*}$ values measured here in visual and GM ROIs (Table 1), however the smaller $\mathrm{R}_{2}{ }^{*}$ measured in the motor ROI means that BOLD contrast in this ROI is $\sim 10 \%$ lower than it would have been if a longer echo time, specifically optimized for the motor ROI, were used. However, since the BOLD contrast is significantly above the noise, this lower BOLD contrast in the motor ROI will have a negligible impact on BOLD sensitivity and will be incorporated into $\mathrm{M}$, not impacting on quantification of $\mathrm{CMRO}_{2}$.

In conclusion, we present a new approach to calibrated BOLD, relaxing the iso-metabolic hypercapnia assumption, whilst measuring the dose-wise change in $\mathrm{CMRO}_{2}$ due to hypercapnia. This approach can map local $\mathrm{CMRO}_{2}$ responses to hypercapnia, so may be suitable for measuring spatial heterogeneity in these responses. This approach may be especially applicable in studies including metabolic pathology, such as diabetes, dementia, and 


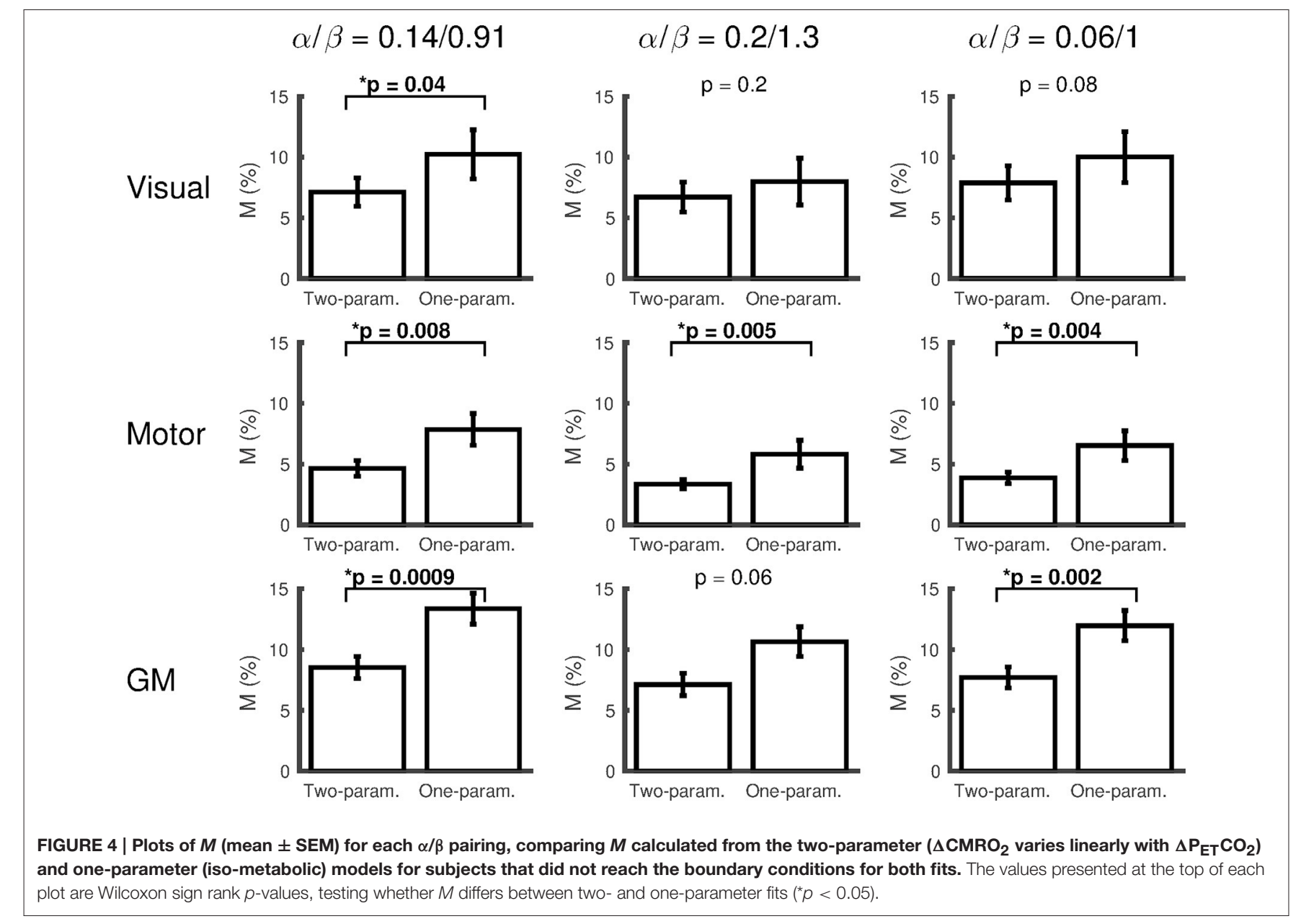

multiple sclerosis, where there may be inter- and/or intra-subject differences in the $\mathrm{CMRO}_{2}$ response to hypercapnia. Further, as well as the calibrated BOLD endpoints of measuring taskdependent $\mathrm{CMRO}_{2}$, or absolute $\mathrm{CMRO}_{2}$, the measurement of the $\mathrm{CMRO}_{2}$ response to hypercapnia could become a tool for investigating cerebral metabolic health in its own right.

\section{AUTHOR CONTRIBUTIONS}

ID and KM developed the idea. KM and RW designed the experiment. KM and RW acquired the data. ID and KM

\section{REFERENCES}

Blockley, N. P., Griffeth, V. E., Stone, A. J., Hare, H. V., and Bulte, D. P. (2015). Sources of systematic error in calibrated BOLD based mapping of baseline oxygen extraction fraction. Neuroimage 122, 105-113. doi: 10.1016/j.neuroimage.2015.07.059

Boxerman, J. L., Hamberg, L. M., Rosen, B. R., and Weisskoff, R. M. (1995). MR contrast due to intravascular magnetic susceptibility perturbations. Magn. Reson. Med. 34, 555-566. doi: 10.1002/mrm.19103 40412 performed data analysis. ID, RW, and KM interpreted the results. ID drafted the manuscript. ID, RW, and KM revised the manuscript.

\section{ACKNOWLEDGMENTS}

The authors wish to thank Ashley Harris for contributing to the data acquisition. ID and KM acknowledge salary support from the Wellcome Trust [WT090199; WT200804]. RW acknowledges the support of the Higher Education Funding Council for Wales (HEFCW).

Bulte, D. P., Kelly, M., Germuska, M., Xie, J., Chappell, M. A., Okell, T. W., et al. (2012). Quantitative measurement of cerebral physiology using respiratory-calibrated MRI. Neuroimage 60, 582-591. doi: 10.1016/j.neuroimage.2011.12.017

Chen, J. J., and Pike, G. B. (2010a). Global cerebral oxidative metabolism during hypercapnia and hypocapnia in humans: implications for BOLD fMRI. J. Cereb. Blood Flow Metab. 30, 1094-1099. doi: 10.1038/jcbfm.2010.42

Chen, J. J., and Pike, G. B. (2010b). MRI measurement of the BOLD-specific flow-volume relationship during hypercapnia and hypocapnia in humans. Neuroimage 53, 383-391. doi: 10.1016/j.neuroimage.2010.07.003 
Chiarelli, P. A., Bulte, D. P., Gallichan, D., Piechnik, S. K., Wise, R., and Jezzard, P. (2007a). Flow-metabolism coupling in human visual, motor, and supplementary motor areas assessed by magnetic resonance imaging. Magn. Reson. Med. 57, 538-547. doi: 10.1002/mrm.21171

Chiarelli, P. A., Bulte, D. P., Piechnik, S., and Jezzard, P. (2007b). Sources of systematic bias in hypercapnia-calibrated functional MRI estimation of oxygen metabolism. Neuroimage 34, 35-43. doi: 10.1016/j.neuroimage.2006.08.033

Croal, P. L., Driver, I. D., Francis, S. T., and Gowland, P. A. (2017). Field strength dependence of grey matter $\mathrm{R}_{2}{ }^{*}$ on venous oxygenation. Neuroimage 146, 327-332. doi: 10.1016/j.neuroimage.2016.10.004

Davis, T. L., Kwong, K. K., Weisskoff, R. M., and Rosen, B. R. (1998). Calibrated functional MRI: mapping the dynamics of oxidative metabolism. Proc. Natl. Acad. Sci. U.S.A. 95, 1834-1839. doi: 10.1073/pnas.95.4.1834

Driver, I., Blockley, N., Fisher, J., Francis, S., and Gowland, P. (2010). The change in cerebrovascular reactivity between $3 \mathrm{~T}$ and $7 \mathrm{~T}$ measured using graded hypercapnia. Neuroimage 51, 274-279. doi: 10.1016/j.neuroimage.2009.12.113

Driver, I. D., Whittaker, J. R., Bright, M. G., Muthukumaraswamy, S. D., and Murphy, K. (2016). Arterial CO2 fluctuations modulate neuronal rhythmicity: implications for MEG and fMRI studies of resting-state networks. J. Neurosci. 36, 8541-8550. doi: 10.1523/JNEUROSCI.4263-15.2016

Dulla, C. G., Dobelis, P., Pearson, T., Frenguelli, B. G., Staley, K. J., and Masino, S. A. (2005). Adenosine and ATP link PCO2 to cortical excitability via pH. Neuron 48, 1011-1023. doi: 10.1016/j.neuron.2005.11.009

Gauthier, C. J., and Hoge, R. D. (2012). Magnetic resonance imaging of resting OEF and CMRO(2) using a generalized calibration model for hypercapnia and hyperoxia. Neuroimage 60, 1212-1225. doi: 10.1016/j.neuroimage.2011.12.056

Germuska, M., Merola, A., Murphy, K., Babic, A., Richmond, L., Khot, S., et al. (2016). A forward modelling approach for the estimation of oxygen extraction fraction by calibrated fMRI. Neuroimage 139, 313-323. doi: 10.1016/j.neuroimage.2016.06.004

Glover, G. H. (1999). Simple analytic spiral K-space algorithm. Magn. Reson. Med. 42, 412-415. doi: 10.1002/(SICI)1522-2594(199908)42:2<412:: AID-MRM25>3.0.CO;2-U

Griffeth, V. E. M., and Buxton, R. B. (2011). A theoretical framework for estimating cerebral oxygen metabolism changes using the calibrated-BOLD method: modeling the effects of blood volume distribution, hematocrit, oxygen extraction fraction, and tissue signal properties on the BOLD signal. Neuroimage 58, 198-212. doi: 10.1016/j.neuroimage.2011.05.077

Grubb, R. L. Jr., Raichle, M. E., Eichling, J. O., and Ter-Pogossian, M. M. (1974). The effects of changes in PaCO2 on cerebral blood volume, blood flow, and vascular mean transit time. Stroke 5, 630-639. doi: 10.1161/01.STR. 5.5 .630

Hall, E. L., Driver, I. D., Croal, P. L., Francis, S. T., Gowland, P. A., Morris, P. G., et al. (2011). The effect of hypercapnia on resting and stimulus induced MEG signals. Neuroimage 58, 1034-1043. doi: 10.1016/j.neuroimage.2011.06.073

Ho, Y. C., Petersen, E. T., Zimine, I., and Golay, X. (2011). Similarities and differences in arterial responses to hypercapnia and visual stimulation. J. Cereb. Blood Flow Metab. 31, 560-571. doi: 10.1038/jcbfm.2010.126

Hoffman, W. E., Miletich, D. J., and Albrecht, R. F. (1982). Cerebrovascular and cerebral metabolic responses of aged rats to changes in arterial PCO2. Neurobiol. Aging 3, 141-143. doi: 10.1016/0197-4580(82)90010-0

Hoge, R. D., Atkinson, J., Gill, B., Crelier, G. R., Marrett, S., and Pike, G. B. (1999). Investigation of BOLD signal dependence on cerebral blood flow and oxygen consumption: the deoxyhemoglobin dilution model. Magn. Reson. Med. 42, 849-863. doi: 10.1002/(SICI)1522-2594 (199911)42:5<849::AID-MRM4>3.0.CO;2-Z

Horvath, I., Sandor, N. T., Ruttner, Z., and McLaughlin, A. C. (1994). Role of nitric oxide in regulating cerebrocortical oxygen consumption and blood flow during hypercapnia. J. Cereb. Blood Flow Metab. 14, 503-509. doi: $10.1038 /$ jcbfm.1994.62

Ito, H., Kanno, I., Ibaraki, M., Hatazawa, J., and Miura, S. (2003). Changes in human cerebral blood flow and cerebral blood volume during hypercapnia and hypocapnia measured by positron emission tomography. J. Cereb. Blood Flow Metab. 23, 665-670. doi: 10.1097/01.WCB.0000067721.64998.F5

Jain, V., Langham, M. C., Floyd, T. F., Jain, G., Magland, J. F., and Wehrli, F. W. (2011). Rapid magnetic resonance measurement of global cerebral metabolic rate of oxygen consumption in humans during rest and hypercapnia. J. Cereb. Blood Flow Metab. 31, 1504-1512. doi: 10.1038/jcbfm.2011.34
Jones, M., Berwick, J., Hewson-Stoate, N., Gias, C., and Mayhew, J. (2005). The effect of hypercapnia on the neural and hemodynamic responses to somatosensory stimulation. Neuroimage 27, 609-623. doi: 10.1016/j.neuroimage.2005.04.036

Kety, S. S., and Schmidt, C. F. (1948). The effects of altered arterial tensions of carbon dioxide and oxygen on cerebral blood flow and cerebral oxygen consumption of normal young men. J. Clin. Invest. 27, 484-492. doi: 10.1172/JCI101995

Kliefoth, A. B., Grubb, R. L. Jr., and Raichle, M. E. (1979). Depression of cerebral oxygen utilization by hypercapnia in the rhesus monkey. J. Neurochem. 32, 661-663. doi: 10.1111/j.1471-4159.1979.tb00404.x

Kudomi, N., Hirano, Y., Koshino, K., Hayashi, T., Watabe, H., Fukushima, K., et al. (2013). Rapid quantitative $\mathrm{CBF}$ and $\mathrm{CMRO}(2)$ measurements from a single PET scan with sequential administration of dual (15)O-labeled tracers. J. Cereb. Blood Flow Metab. 33, 440-448. doi: 10.1038/jcbfm.2012.188

Kwong, K. K., Belliveau, J. W., Chesler, D. A., Goldberg, I. E., Weisskoff, R. M., Poncelet, B. P., et al. (1992). Dynamic magnetic resonance imaging of human brain activity during primary sensory stimulation. Proc. Natl. Acad. Sci. U.S.A. 89, 5675-5679. doi: 10.1073/pnas.89.12.5675

Lammertsma, A. A., and Jones, T. (1983). Correction for the presence of intravascular oxygen-15 in the steady-state technique for measuring regional oxygen extraction ratio in the brain: 1. Description of the method. J. Cereb. Blood Flow Metab. 3, 416-424. doi: 10.1038/jcbfm.1983.67

Lammertsma, A. A., Wise, R. J., Heather, J. D., Gibbs, J. M., Leenders, K. L., Frackowiak, R. S., et al. (1983). Correction for the presence of intravascular oxygen-15 in the steady-state technique for measuring regional oxygen extraction ratio in the brain: 2. Results in normal subjects and brain tumour and stroke patients. J. Cereb. Blood Flow Metab. 3, 425-431. doi: $10.1038 /$ jcbfm. 1983.68

Leontiev, O., and Buxton, R. B. (2007). Reproducibility of BOLD, perfusion, and CMRO2 measurements with calibrated-BOLD fMRI. Neuroimage 35, 175-184. doi: 10.1016/j.neuroimage.2006.10.044

Liu, T. T., Wong, E. C., Frank, L. R., and Buxton, R. B. (2002). Analysis and design of perfusion-based event-related fMRI experiments. Neuroimage 16, 269-282. doi: 10.1006/nimg.2001.1038

Lu, H., Zhao, C., Ge, Y., and Lewis-Amezcua, K. (2008). Baseline blood oxygenation modulates response amplitude: physiologic basis for intersubject variations in functional MRI signals. Magn. Reson. Med. 60, 364-372. doi: $10.1002 / \mathrm{mrm} .21686$

Mark, C. I., Fisher, J. A., and Pike, G. B. (2011). Improved fMRI calibration: precisely controlled hyperoxic versus hypercapnic stimuli. Neuroimage 54, 1102-1111. doi: 10.1016/j.neuroimage.2010.08.070

Merola, A., Murphy, K., Stone, A. J., Germuska, M. A., Griffeth, V. E., Blockley, N. P., et al. (2016). Measurement of oxygen extraction fraction (OEF): an optimized BOLD signal model for use with hypercapnic and hyperoxic calibration. Neuroimage 129, 159-174. doi: 10.1016/j.neuroimage.2016.01.021

Mintun, M. A., Raichle, M. E., Martin, W. R., and Herscovitch, P. (1984). Brain oxygen utilization measured with $\mathrm{O}-15$ radiotracers and positron emission tomography. J. Nucl. Med. 25, 177-187.

Murphy, K., Harris, A. D., and Wise, R. G. (2013). "Measuring the influence of hypercapnia on absolute CMRO2 in humans," in Proceedings ISMRM, (Salt Lake City, UT), 3342.

Noth, U., Meadows, G. E., Kotajima, F., Deichmann, R., Corfield, D. R., and Turner, R. (2006). Cerebral vascular response to hypercapnia: determination with perfusion MRI at 1.5 and 3.0 Tesla using a pulsed arterial spin labeling technique. J. Magn. Reson. Imaging 24, 1229-1235. doi: 10.1002/ jmri.20761

Ogawa, S., Menon, R. S., Tank, D. W., Kim, S. G., Merkle, H., Ellermann, J. M., et al. (1993). Functional brain mapping by blood oxygenation level-dependent contrast magnetic resonance imaging. A comparison of signal characteristics with a biophysical model. Biophys. J. 64, 803-812. doi: 10.1016/S0006-3495(93)81441-3

Ogawa, S., Tank, D. W., Menon, R., Ellermann, J. M., Kim, S. G., Merkle, H., et al. (1992). Intrinsic signal changes accompanying sensory stimulation: functional brain mapping with magnetic resonance imaging. Proc. Natl. Acad. Sci. U.S.A. 89, 5951-5955. doi: 10.1073/pnas.89.13.5951

Rhodes, C. G., Lenzi, G. L., Frackowiak, R. S., Jones, T., and Pozzilli, C. (1981). Measurement of CBF and CMRO2 using the continuous inhalation of $\mathrm{C} 15 \mathrm{O} 2$ 
and $15 \mathrm{O}$. Experimental validation using $\mathrm{CO} 2$ reactivity in the anaesthetised dog. J. Neurol. Sci. 50, 381-389. doi: 10.1016/0022-510X(81)90150-7

Sicard, K. M., and Duong, T. Q. (2005). Effects of hypoxia, hyperoxia, and hypercapnia on baseline and stimulus-evoked BOLD, CBF, and CMRO2 in spontaneously breathing animals. Neuroimage 25, 850-858. doi: 10.1016/j.neuroimage.2004.12.010

Ter-Pogossian, M. M., and Herscovitch, P. (1985). Radioactive oxygen-15 in the study of cerebral blood flow, blood volume, and oxygen metabolism. Semin. Nucl. Med. 15, 377-394. doi: 10.1016/S0001-2998(85)80015-5

Wise, R. G., Harris, A. D., Stone, A. J., and Murphy, K. (2013). Measurement of OEF and absolute CMRO2: MRI-based methods using interleaved and combined hypercapnia and hyperoxia. Neuroimage 83, 135-147. doi: 10.1016/j.neuroimage.2013.06.008

Wong, E. C., Buxton, R. B., and Frank, L. R. (1998). Quantitative imaging of perfusion using a single subtraction (QUIPSS and QUIPSS II). Magn. Reson. Med. 39, 702-708. doi: 10.1002/mrm. 1910390506

Xu, F., Uh, J., Brier, M. R., Hart, J. Jr., Yezhuvath, U. S., Gu, H., et al. (2011). The influence of carbon dioxide on brain activity and metabolism in conscious humans. J. Cereb. Blood Flow Metab. 31, 58-67. doi: 10.1038/jcbfm.2010.153
Yablonskiy, D. A. (2011). Cerebral metabolic rate in hypercapnia: controversy continues. J. Cereb. Blood Flow Metab. 31, 1502-1503. doi: 10.1038/jcbfm. 2011.32

Zappe, A. C., Uludag, K., Oeltermann, A., Ugurbil, K., and Logothetis, N. K. (2008). The influence of moderate hypercapnia on neural activity in the anesthetized nonhuman primate. Cereb. Cortex 18, 2666-2673. doi: 10.1093/cercor/ bhn023

Conflict of Interest Statement: The authors declare that the research was conducted in the absence of any commercial or financial relationships that could be construed as a potential conflict of interest.

Copyright (c) 2017 Driver, Wise and Murphy. This is an open-access article distributed under the terms of the Creative Commons Attribution License (CC $B Y)$. The use, distribution or reproduction in other forums is permitted, provided the original author(s) or licensor are credited and that the original publication in this journal is cited, in accordance with accepted academic practice. No use, distribution or reproduction is permitted which does not comply with these terms. 